or metabolic conditions ${ }^{2}$.

It is not clear how these results will compare to the outcome of mitochondrial replacement in human babies. "Whatever we learn in a person will be completely new," Egli says.

\section{ADVISE AND CONSENT}

The answers are not likely to come from the child born in Zhang's clinic. The study says that the baby's parents have refused any further mitochondrial testing of their child unless there is a medical need. It is not clear whether the family was ever asked to consent to longterm medical monitoring; Zhang and New Hope did not respond to Nature's request for comment on the issue.

The value of the experiment will be limited if scientists cannot track the boy as he grows, Egli says. "It looks like a rush to use this as a treatment and telling patients that this is the treatment, during a time when we still know very little about what the outcomes are."

Guidelines for human research generally require that people be allowed to withdraw from experiments. When this happens, it can make it hard to determine whether a treatment is safe, says Alta Charo, a bioethicist at the University of Wisconsin-Madison. In this case, she says, it is unclear whether the parents received enough information to appreciate how long-term follow-up could

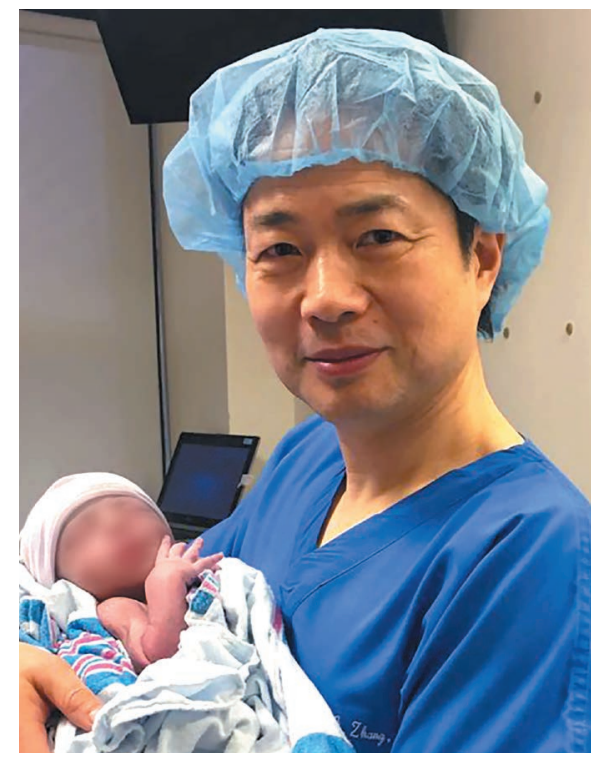

John Zhang holds the baby boy in his clinic.

benefit their child as well as science.

A three-page editorial ${ }^{3}$ accompanying the study notes that the researchers asked the baby's parents to sign a consent form acknowledging that their egg was undergoing an experimental technique. But the form only described the procedure superficially, and did not inform the couple of the potential risks of using this method to create a child.

Because the boy could not give consent, "the duties were even higher for clinicians and participants to protect the best interest of the future child", says Rosario Isasi, a legal scholar at the University of Miami in Florida. In his paper, Zhang says that the parents received $\frac{3}{z}$ "cautious counselling for mitochondrial replacement therapy".

He told Nature last week that his team will continue to test the technique, using eggs from prospective mothers who are between 42 and 47 years of age. They want to explore whether mitochondria from younger donors' cells may stimulate the older eggs' ability to be fertilized and develop normally.

1. Zhang, J. et al. Reprod. Biomed. Online 34, 361-368 (2017).

2. Wallace, D. C. et al. Cold Spring Harb. Perspect. Biol.. 5, a021220 (2013)

3. Alikani, M., Fauser, B. C. J., Garcia-Valesco, J. A., Simpson, J. L. \& Johnson, M. H. Reprod. Biomed. Online 34, 333-368 (2017).

\section{CORRECTION}

The News Feature 'How to hunt for a black hole' (Nature 543, 478-480; 2017) erred in saying that Heino Falcke led a team that made one of the first VLBI observations. He was one of the team's investigators. 